\title{
WIMU: WEARABLE INERTIAL MONITORING UNIT a MEMS-based device for swimming performance analysis
}

\author{
Ana S. Silva, Miguel F. Correia \\ Institute for Systems and Computer Engineering of Porto (INESC Porto), Rua Dr. Roberto Frias, 378, Porto, Portugal \\ amsilva@inescporto.pt, miguel.velhote.correia@inescporto.pt \\ Antonio J. Salazar, Carla M. Borges \\ Faculdade de Engenharia da Universidade do Porto (FEUP), University of Porto, Porto, Portugal \\ dee08011@fe.up.pt,deb09010@fe.up.pt
}

Keywords: Wearable, monitoring device, biomechanical parameters, MEMS, swimming analysis.

Abstract: Advances in sensor technology, electronic textile integration, and integrated circuits have introduced a paradigm shift in the way most researchers approach signal monitoring. In recent years, devices such as body sensor networks (BSN) allow for direct on-body physiological and biomechanical parameters measurements. Such technology allows for a more in depth analysis of an athlete's performance, without affecting the results due to awkward wires or uncomfortable carry-on devices. Miniaturization and other achievements allow a more seamless interaction with the individual, permitting a more natural behaviour during the monitoring session. The project BIOSWIM (Body Interface System based on Wearable Integration Monitorization) is a joint multidisciplinary effort of a number of Portuguese universities which seeks a pervasive monitoring solution for performance, physiological and biomechanical signals from a swimmer under normal training conditions. In order to achieve such an undertaking a swimsuit prototype was developed with truly integrated EKG textile sensors; which will work in conjunction with a wearable inertial monitoring unit (WIMU) and a wearable chemical monitoring unit. This article focuses on the WIMU, which serves as the biomechanical data processing unit of the system.

\section{INTRODUCTION}

Words such as ubiquitous, pervasive, ambient, and seamless are common among most technological research areas of our day; sport is no exception. In order to evaluate an athlete's performance, coaches and trainers have been dependent on visual markers and inferred biomechanical data for years. Until recently, high performance inertial sensors' prohibitorily high cost excluded them from cost sensitive areas. Nowadays, the development of miniaturized low-powered accelerometers and gyroscopes allow their inclusion on wearable monitoring alternatives, closing the gap between the athlete's perception and that of his/her trainers. In fact recent advances, such as microelectromechanical systems (MEMS), have introduced a viable alternative, which unlike many of its counterparts; can be made to be wearable and water friendly, as to be used on swimming analysis. Signals such as instantaneous velocity, stroke frequency, and angular positions are key elements for feature extraction and pattern recognition strategies.

There are multiple paradigms shifts taking place, were the single moment is being replace by continuous long-term data gathering (instead of a doctor's consult, a patient's data can be gathered for days or weeks); the laboratory or controlled environments is being replaced by real-life scenarios (individuals can interact with their natural surroundings, such as "in vitro" experiments lack the depth of "in vivo" experiments); and database analysis is being replaced (although more complete and complex databases are also emerging) by realtime analysis (where data gathering can change an athlete's strategy mid-event). These conceptual changes are accompanied by a new group of concepts, methodologies and technologies. It has been with advances in integrated circuits (IC), wireless sensor network (WSN), signal processing, etc., in a truly multidisciplinary effort (electrical, 
biomedical, medicine, textile, among others), that never before has the world seen so many systems designed for monitoring an individual's performance and reactions, as in the last decade.

Although the wearable inertial monitoring unit (WIMU) is still at a prototype stage, and is intended to follow a BSN-like methodology at its final stage, preliminary data reveals the promise of such a device.

\section{BIOSWIM PROJECT}

Electronic textiles, interactive textiles, smart garments and wearables are terms expressing one of the most recent research trends in textile science and technology. From late 1990's until now investigators from research centers and companies proposed several approaches and solutions for many different problems, from technical applications to leisure. While the fastest developments started with multimedia applications, very soon the scientific community understood the potential of these technologies on other areas, like in medicine and sports. Literature describes a large number of projects intended to remotely measure physiological parameters on mainly heart failure patients, such as MyHeart and Wealthy projects (Pacelli, 2006), VTAMN (Noury, 2004) and more recently BIOTEX (Coyle, 2010) and Vital Jacket (Cunha, 2010).

The BIOSWIM project is inspired in all these emerging technologies and intends to apply them in a smart garment which can allow the measurement of vital signs and other significant signals both for health and particularly for sport athletes, thus contributing for more knowledge in these areas. The wearable equipment combining comfort properties together with sensing devices will fit on an individual in an optimal way without the need of using unpleasant methods for fixing sensors on the athletes. Electromyography, cardiac rhythm, respiratory effort, oxygen consuming taxes, motion capture, wrist and arm accelerations and rotations, speed, hydrodynamics' pressure constitute some of the parameters of interest of this research project and intended to be collected at simultaneous time using the same wearable, thus consisting in a major breakthrough in the sport sciences (see Figure 1). This project is a multidisciplinary effort of three major Portuguese institutions: Centre of Textile Science and Technology of the University of Minho, INESC Porto (Institute for Systems and Computers Engineering of Porto) and the Faculty of Sports of the University of Porto.

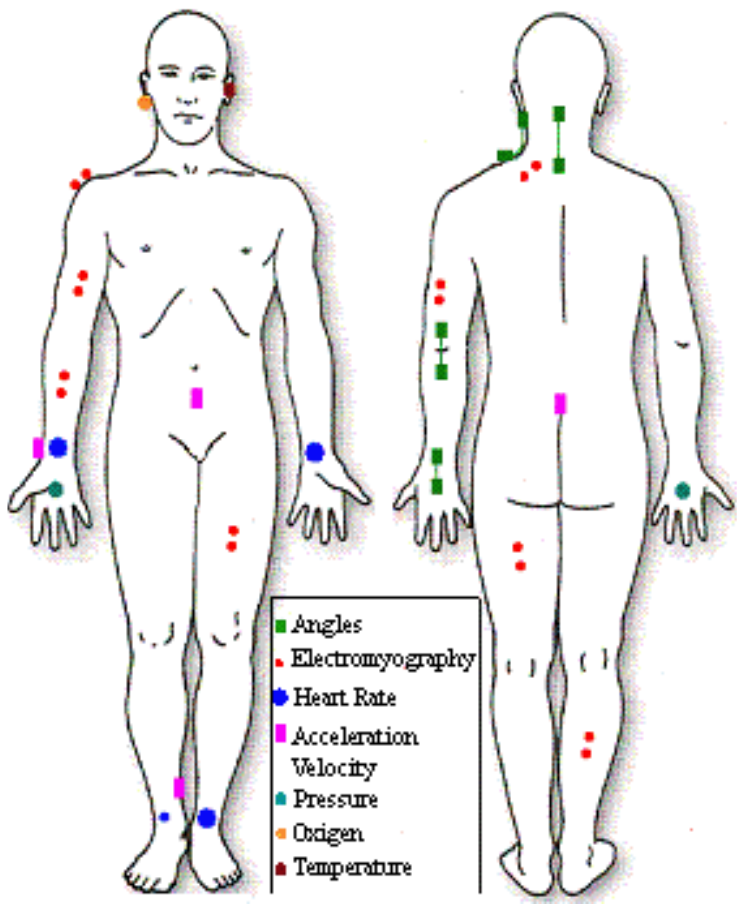

Figure 1: BIOSWIM project's monitoring objectives

The main goals of the BIOSWIM are the development of wearable prototypes for measuring several biological parameters, critical for sport performance and health purposes, oriented for regular sport and for in-pool (or underwater depending of the sports) activity. Moreover, the wearable must be design with maximum comfort, comprehending the best combination of textile material with embedded microelectronics for signal acquisition and data transmission. Also, an universal electronic system for power supply, data acquisition, processing, management, and communication working for both inside and outside water environments and embedded on textile substrate is expected to be developed.

An immediate application of this wearable is in sports, for competition athletes, sport amateurs, and body maintenance. While for the less demanding activities some of the actual systems provide the essential information, the same may not apply when it is intended to evaluate performance on athletes. The main goal in this situation is the improvement of their performance in order to achieve better results. Proper movement inside water will definitely contribute for higher speed. Beyond vital signs, other signals are crucial for optimizing the athlete effort and thus reduce the human fatigue when subjected to intensive training or competition charges.

The development of wearable monitoring devices for sports practitioners can present several 
noticeable repercussions in sportive community, both with reference to the optimization of the training process of elite athletes, as well as to the promotion of safety in rehabilitation and leisure sports. The idea behind the BIOSWIM project is to produce an elite training evaluation wearable station, allowing the most innovative monitoring, ambulatory registration, real-time visualization and post-exercise display of both physiological and biomechanical relevant data for training (heart-rate, respiratory frequency, oesophageal temperature, sweat, arm tri-axial acceleration, body vertical acceleration).

Important repercussions are expected both for practitioners, coaches and scientists, allowing an increased safety in physical activity, an augmented objectivity - and efficiency - of the elite training process, and an easy data collection for scientific research in neuro-physiology and biomechanics of sport.

\section{ARCHITECTURE OVERVIEW}

Whilst a trend seems to be forming within wearable monitoring systems (WMS), for a preference of commercially available monitoring solutions, referred to as "motes" by some (which tend to be the result of past efforts in wireless sensor networks). There is also a necessity for custom designs which seek to optimize the data collection of the particular project at hand. There seems to be some concurrence on the general design of a body sensor network (BSN) (Akyildiz, 2002) (Tufail, 2009) (Pantelopoulos, 2010), or in this case a WMS (as the particular functional objective). Commercially available "motes" are commonly found as the main elements of BSN and WMS designs, some examples are: IRIS, MICAz, TELOSb, SHIMMER and Imote2. These devices are used for capturing and transmitting biomechanical and physiological signals, among other data related to healthcare, sports, motion capturing, etc. These off-the-shelf solutions are a considerable contribution to the field of BSN and WMS, since they permit research groups (specially those that are not electronics inclined) to focus on the captured data and feature extraction algorithms/heuristics, instead of the, sometimes slow process of electronics debugging and troubleshooting process.

Within this article, monitoring systems (MS) will be considered divided in three main sections:

- Sensing section.

- Processing section.
- Transmitting section.

The mentioned sections can be found separate, intermixed or integrated depending on the design, but the objectives of their functions can be readily separated if needs be.

\subsection{Sensing section}

Advances in micro-fluidics, material science, nanostructures, micro-electromechanical devices, bioelectrical interfaces, and others; have contributed to a new generation of wearable and implantable sensors and monitoring devices. Healthcare has greatly benefitted from the development of biosensors (also referred to as chemical sensors) (Patel, 2006) and physiological sensors. Such achievements have paved the way for truly pervasive monitoring strategies, which will benefit patients and reduce the load to health-care facilities. From a sport monitoring perspective, non-invasive, minimally intrusive sensors are the preferred choice, and consideration of their positioning, calibration, noise, offset, deviation, etc., are concerns (Yang, 2007). There exist a wide array of commercially available sensors and even more experimental devices and concepts waiting their turn.

\subsection{Processing section}

In today's market, the competition to claim to be the lowest powered microcontroller is fierce. Depending of the complexity required by the application and the feature extraction methods to be applied, an array of Reduced Instruction Set Computer (RISC) or Advanced RISC Machine (ARM) architecture based microcontrollers offer different features which accommodate varying solutions. Based on the popular "motes" designs and further research in the area (unpublished work from the authors), there seems to be a preference for Texas Instrument MSP430 ultra-low power, Atmel's AVMEGA ultralow power, and the Microchip's extreme-low-power (XLP) PIC microcontrollers. Using the power specifications, indicated on the datasheet of each microcontroller, as a base for comparison, can sometimes lead to problems and confusion; careful attention must be paid to the conditions in which each manufacturer measures their devices power consumption. 


\subsection{Transmitting Section}

When referring to WMS, it is unavoidable to consider a wireless component for interfacing with the system; either be it for real-time (or continuous) or sporadic updating to a remote processing node, or for downloading the collected stored data, or even for transmitting the data from a sensor node to the on-body or remote processing unit. The presence of cables or the need for physical removal of the device for data download represents an alternative that while permissible at prototyping and troubleshooting stages, is impractical at more advance stages of design and implementation.

A number of alternatives exist for mid-range wireless communication including common protocols (GSM, WiMAX, UMTS, WLAN, etc.) and upcoming $4 \mathrm{G}$ mobile communication solutions. From a more local point of view the IEEE 802.15 Workgroup has introduced and arrays of solutions. Among the favorite standards one counts with the IEEE 802.15.1, known as Bluetooth, and the IEEE 802.15.4, also referred to as Zigbee. The number of low-power short-range transceivers in the market today is enough to overwhelm even experienced researchers. It seems every brand offers their particular RF solution, claiming low-power transmission; companies such as Texas Instrument, Atmel, Semtech, Maxim and Microchip (to mention a few), offer interesting and varying solutions.

\section{WIMU}

For years, numerous devices and setups have been implemented in order to assist on swimming performance analysis. Many of these devices were based on video analysis, while others made direct measurement and signal capturing through awkward setups, generally uncomfortable for the swimmer and thus affecting hers/his performance. Advances in a number of fields have allowed for compact wearable monitoring devices, greatly improving the data gathering process and closing the gap for a truly seamless biomechanical signal monitoring solution. Although there is a relatively reduced number of biomechanical signal monitoring systems being used for swimming performance analysis today (particularly when compared to the number of wearable monitoring devices for healthcare or even for land based sports), a shift on the approaches for swimming analysis is being noted. Different strategies have been applied by the mentioned systems, however a common element seem to be their dependence on accelerometers. Some systems worth mentioning are the ETH Zurich Wearable Computing Laboratory's SwimMaster (Bächlin, 2009) and Imperial College BSN device (Pansiot, 2010).

\subsection{Design Overview}

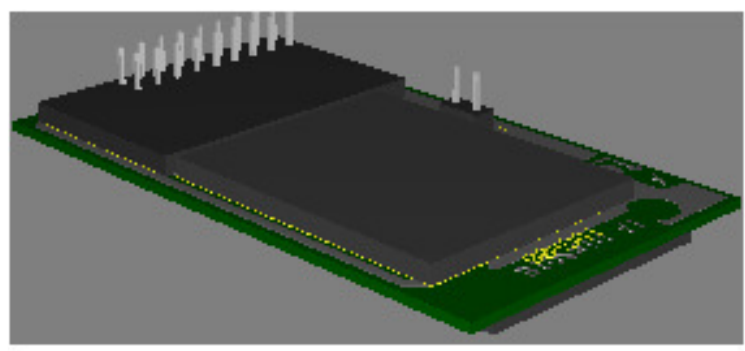

(a)

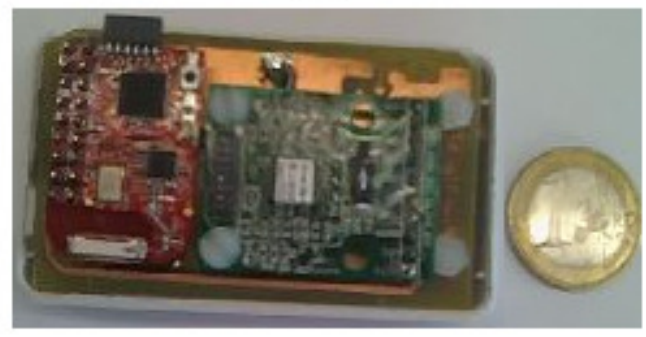

(b)

Figure 2: (a) Tri-dimensional view of the WIMU. (b) Top view of the system.

The WIMU - Wearable Inertial Measurement Unit was designed in the scope of the BIOSWIM project. The goal was to develop a MEMS-based wearable device for assessing biomechanical parameters of a swimming athlete. The inertial unit comprises a triaxial accelerometer for linear acceleration and a gyroscope for angular velocity measurements. In addition, a microcontroller for signal acquisition, conversion and wireless transmission is used. A 3D schematic and a picture of the overall system are depicted in Figure 2.

Both inertial sensors used are MEMS devices. Micromachined inertial sensors have a huge potential for applications in the biomedical field. They fulfill the requirements of small volume, portability, low power consumption and they are available at low cost

To extract the acceleration value, the accelerometer has a movable mass which is connected to a fixed frame via spring structures. An external acceleration will displace the mass from its rest position, proportionately to the input movement. The micromachined gyroscope used relies on a mechanical structure that is driven into resonance 
and excites a secondary oscillation in either the same structure or in a second one, due to Coriolis force. The amplitude of this secondary oscillation is directly proportional to the angular rate signal to be measured.

Commercially available evaluations boards were used to integrate the MEMS sensors into the WIMU. The accelerometer evaluation board is a small circuit board intended to be used for evaluating the MMA7260QT accelerometer and developing prototypes quickly without requiring a PCB to be designed. It also provides means for understanding the best mounting position and location of this accelerometer. Similarly, the IDG-300 gyroscope is integrated in an evaluation board along with the electronics necessary for application-ready functionality

In order to integrate both the accelerometer and the gyroscope into the inertial unit, a microcontroller was selected from the many commercially available devices. The one chosen is the eZ430-RF2500 development tool from Texas Instrument. The eZ430-RF2500 uses the MSP430F2274 microcontroller which combines 16-MIPS performance with a 200-ksps 10-bit ADC and 2 opamps and is paired with the CC2500 multi-channel radio-frequency $(\mathrm{RF})$ transceiver designed for lowpower wireless applications. This board is a complete wireless development tool that includes all the hardware and software required to develop an entire wireless project. One reason for choosing this board is its low-power consumption characteristic.

The wireless communication uses the SimpliciTI protocol which belongs to Texas Instruments and is a low-power RF $(2.4 \mathrm{GHz})$ protocol aimed for simple and small RF networks. It was designed for easy implementation with minimal microcontroller resource requirements.

The WIMU behaves like an End Device in a sensor network topology. It is responsible for acquisition and conversion of the sensor signals and posterior wireless transmission to a remote station for post-processing and analysis. It was designed so that multiple WIMUs could be used in different body segments in a truly body sensor network.

\subsection{WIMU Architecture}

As mentioned above, the WIMU consists of a 3axial accelerometer, a gyroscope, a microcontroller and a power supply unit. The architecture of the WIMU is represented in Figure 3.

The acceleration and angular velocity signals are acquired and converted sequentially by the 10-bit ADC integrated in the MSP430F2274 microcontroller at a sampling frequency of approximately
50 Ksps. A time-stamp is then retrieved from the system and added to a sensor packet containing the 3 -axis accelerations and angular velocity. Therefore, the final packet frame consists of sensor signals together with a time-stamp, which is then ready to be sent by the CC2500 radio frequency to a remote station where the sensor data can be analysed and processed.

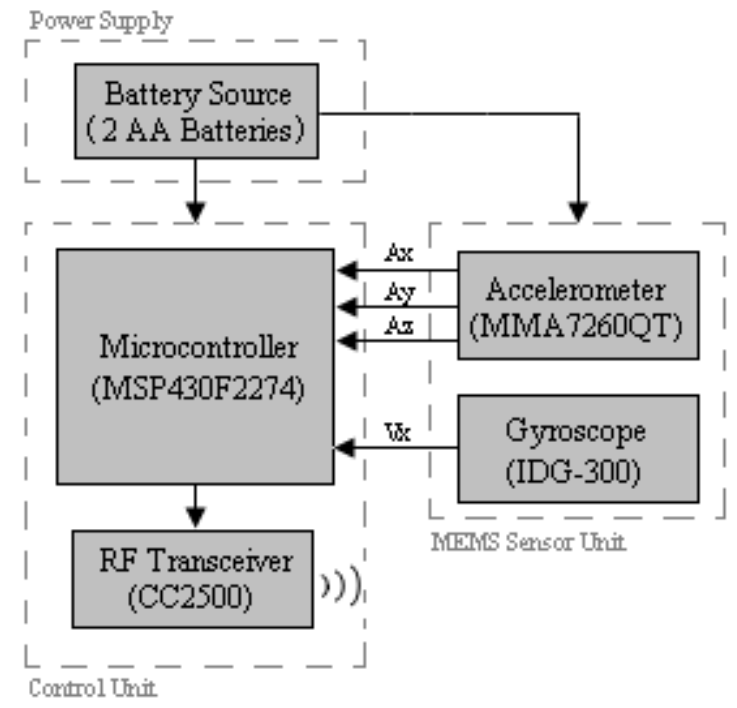

Figure 3: WIMU architecture

The WIMU weights approximately 65.6 grams and measures $57 \times 90.5 \times 24 \mathrm{~mm}$. This way the device can unobtrusively be worn by the swimmer without affecting his/her performance during swimming. With two AA batteries the system can operate continuously up to four hours.

\section{EXPERIMENTAL RESULTS}

In this study, the sensor was placed on the upper back of the swimmer as can be seen on Figure 4. Before entering the pool, the swimmer was asked to perform a number of flexibility related movements routines in order to determine if she felt movement constraints. Once in the pool, she was asking to swim, submerge and perform various movements in order to asses if the WIMU's presence represented an obstruction to her movements. In both cases (outside and inside the pool) the swimmer reported that the unit did not affect her movements in any manner. Several more fast submersions and from wall impulses were performed as to determine the waterproofness of the package, proving to function adequately. Finally, the swimmer was told to complete several sets of laps using free-style (i.e. 


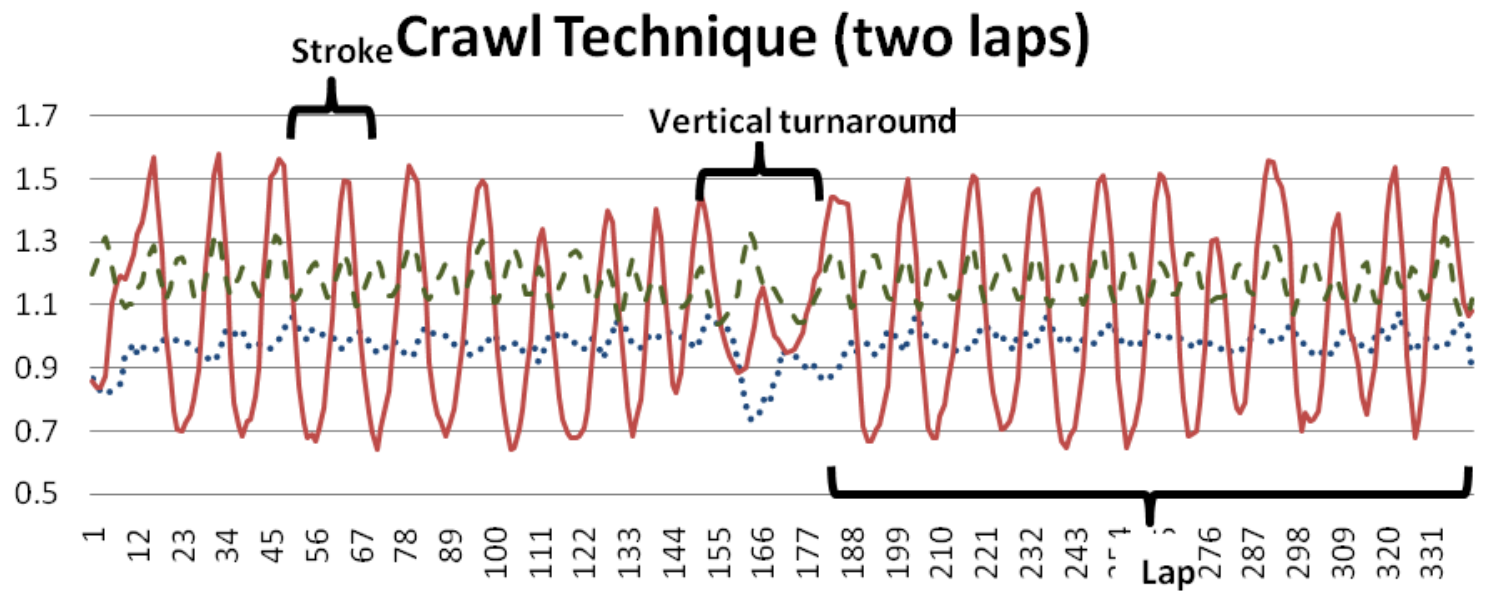

Figure 5: Two laps using Crawl technique signals

crawl) technique, then a number of laps with breaststroke, and finally butterfly. For all styles indicated the end of pool turn was perform through a vertical turnaround (i.e., stop-touch wallturnaround), reversing direction without flipping under water (in order to avoid signal loss).

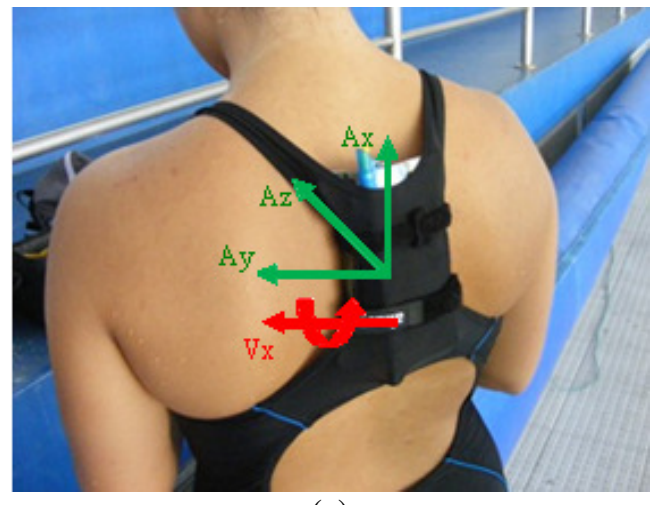

(a)

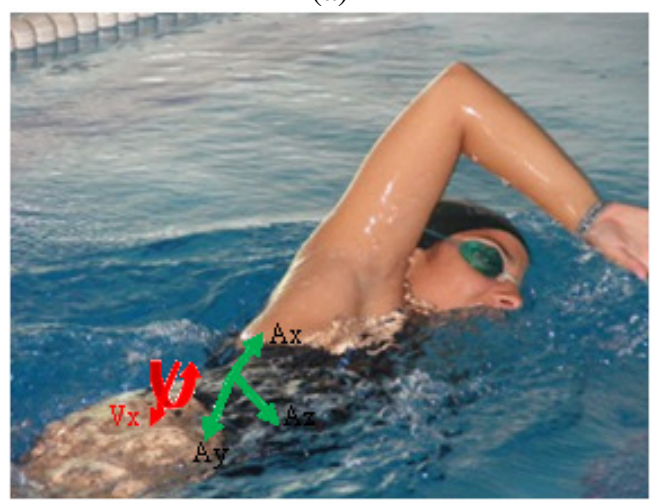

(b)

Figure 4: a) WIMU positioned at the upper back of the athlete. b) The athlete swimming with the WIMU

\section{Crawl Technique}

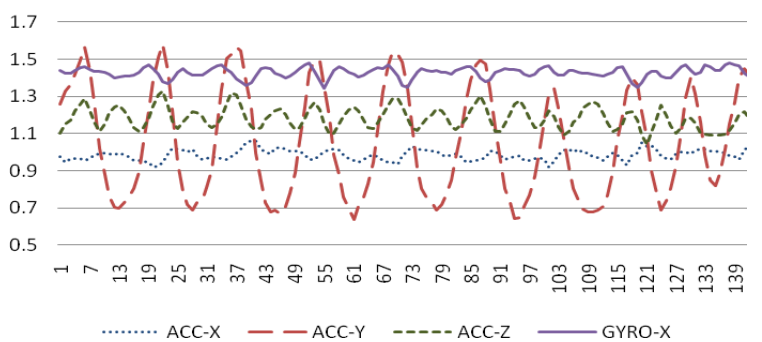

Butterfly Technique

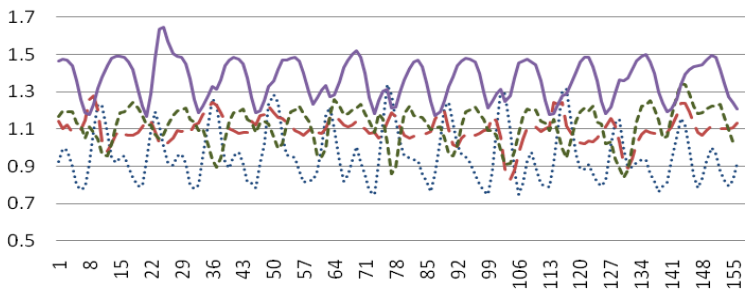

........ ACC-X — ACC-Y -----ACC-Z G GRO-X

Breaststroke Technique

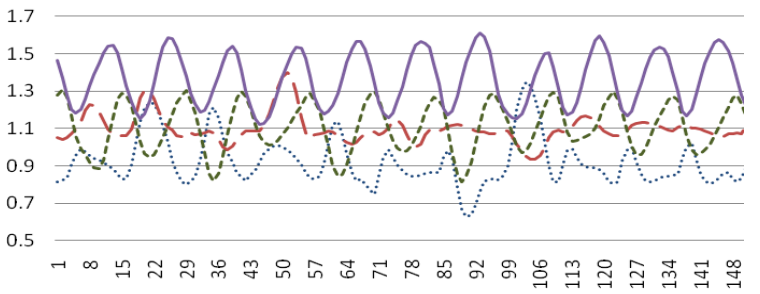

........ ACC-X —-ACC-Y --.--ACC-Z - GYRO-X

Figure 6: One lap captured signals for each performed technique

The signals are captured and buffered, and although there is the potential for signal conditioning at this point, the raw data is sent to the base station 
as is. Synchronization between the base station and the WIMU allow for missed data marking, in order to apply signal restoration strategies. Such data is compensated through cubic interpolation when necessary and then smoothed by a convolution approach. At this point, the crawl technique requires of no compensation (i.e., interpolation) for missed data points since no link loss occurs for a significant period (except for horizontal flip at the end of a pool lap). Although the butterfly and breaststroke technique do present gaps due to momentary loss of signal they do not represent a significant number of points as to deteriorate the signals core; some resulting data can be observed on Figures 5 and 6 .

Observing the graphs concerning each swimming technique, it was possible to differentiate between styles, each stroke, and end of pool. For example, free style can be readily distinguish from breaststroke and butterfly by the signal provided by the accelerometer in $\mathrm{Y}$-axis. The referred signal presents large variations for the crawl technique, while for the other presented styles these variations are comparatively small. Alternatively, the butterfly and breaststroke technique can be differentiate from the data produced by the accelerometers in the $\mathrm{X}$ and $\mathrm{Z}$ axes; which in the case of the butterfly samples they are both almost double the frequency when compared to the angular velocity. In the meanwhile the breaststroke samples show all these three signals at nearly equal frequency. It is still early in this project to produce conclusive results regarding feature extraction for performance analysis, and there is a clear need for accumulating data of diverse swimmers of different genders and competitive level, however these first steps are quite promising. A more comprehensive analysis of the data gathered up to now and future collections, as well as feature extraction strategies for performance analysis will be presented in future works.

\section{CONCLUSIONS AND FUTURE WORK}

In this paper, we present a prototype for swimming performance analysis monitoring based on accelerometers and gyroscopes, referred to as WIMU. The WIMU is one of the objectives of a much more ambitious multi-disciplinary effort of a group of Portuguese universities known as the BIOSWIM project. Such project seeks to characterize swimming through physiological and biomechanical signal capturing at points distributed throughout the athlete's body. Although the device presented is still at the initial stages of development it was capable of providing promising data under inpool normal conditions. The current version of the WIMU serves as a basis for future implementation that will focus on wearability, energy harvesting, and integration within the BIOSWIM's project swimsuits.

\section{ACKNOWLEDGEMENTS}

The authors would like to acknowledge the Foundation for Science and Technology of Portugal for their support of the BIOSWIM project (PTDC/EEA-ELC/70803/2006) and of some of the $\mathrm{PhD}$ students involved in this article (SFRH/BD/61396/2009 and SFRH/BD/60929 12009). Additionally the authors would like to acknowledge the contribution of Barbara Mota, main testing swimmer.

\section{REFERENCES}

Akyildiz, L., Su, W., Sankarasubramaniam, Y., Cayirci, E. 2002. Wireless Sensor Networks: a survey. In Computer Network.vol. 38, issue 4, pp. 393-422.

Bächlin, M., Förster, K., Tröster, G., 2009. SwimMaster: a wearable assistant for swimmer. In $11^{\text {th }}$ International Conference on Ubiquitous computing. ACM. pp. 215224.

Coyle, S., K.-T. Lau, et al. 2010. BIOTEX - Biosensing Textiles for Personalised Healthcare Management. In IEEE Transactions on Informations Technology in Biomedicine 14(2): 364-370.

Cunha, J. P., B. Cunha, et al. 2010. Vital-Jacket: A wearable wireless vital signs monitor for patients' mobility in cardiology and sports. In 4th International Conference on Pervasive Computing Technologies for Healthcare (PervasiveHealth).

Noury, N., A. Dittmar, et al. 2004. VTAMN - A Smart Clothe for Ambulatory Remote Monitoring of Physiological Parameters and Activity. In 26th Annual International Conference of the IEEE Engineering in Medicine and Biology Society, IEMBS '04. IEEE Conference.

Pacelli, M., G. Loriga, et al. 2006. Sensing Fabrics for Monitoring Physiological and Biomechanical Variables: E-textile solutions. In 3rd IEEE/EMBS International Summer School on Medical Devices and Biosensors. IEEE Conference.

Pansiot, J., Lo, B., Yang, G. Z., 2010. Swimming Stroke Kinematic Analysis with BSN. In 2010 International Conference on Body Sensor Networks. IEEE Conference. pp. 153-158.

Pantelopoulos, A., Bourbakis, N. 2010. A survey on wearable sensor-based systems for health monitoring 
and prognosis. In IEEE Transaction on Systems, man \& cybernetics, vol. 20, n. 1, pp. 1-12.

Patel, B., Anastassiou, C., O'Hare, D. 2006. Biosensor Design and Interfacing. In Body Sensor Networks, G. Z. Yang, Ed. Springer London, pp. 41-87.

Tufail, F., Islam, H., 2009, Wearable Wireless Body Area Networks. In International Conference on Information Management and Engineering. IEEE Conference.

Yang, L., Shouqian, S. 2007. Smart Sport Underwear Design. In 7th International Conference on Computer Aided Industrial Design \& Conceptual Design, pp. 13. 